\title{
Rastreamento populacional para o câncer de próstata: mais riscos que benefícios
}

\author{
| ${ }^{1}$ Ricardo Ewbank Steffen, ${ }^{2}$ Anete Trajman, ${ }^{3}$ Marisa Santos, ${ }^{4}$ Rosângela Caetano |
}

Resumo: Recentemente, inúmeras campanhas nacionais promovidas por hospitais, sociedades médicas e outras organizaçóes têm estimulado o rastreamento do câncer de próstata, em consonância com iniciativas mundiais conhecidas como Novembro Azul. Essas campanhas aconselham a utilização do toque retal acompanhado da dosagem sérica do antígeno prostático específico em faixas etárias definidas. A motivação seria a detecção precoce da neoplasia, com redução de sua mortalidade e das complicaçóes e impactos associados ao seu tratamento. A dosagem do PSA para fins de rastreamento é alvo de grande controvérsia, visto que a maioria dos tumores detectados pelo rastreamento é de evolução lenta e náo interfeririam na sobrevida ou na qualidade de vida do paciente. $\mathrm{O}$ rastreamento de base populacional não é a indicação de inúmeras instituiçóes estrangeiras e, no Brasil, o Instituto Nacional de Câncer também não recomenda à organização programas de rastreamento desse tipo. $\mathrm{O}$ artigo discute os riscos e benefícios associados a esse tipo de estratégia e reforça a preocupaçáo com o uso inadequado e indiscriminado do rastreamento para o câncer de próstata.

> Palavras-chave: neoplasias da próstata; programas de rastreamento; antígeno prostático específico; sobrediagnóstico.

\author{
1 Instituto de Medicina Social, \\ Universidade do Estado do Rio de \\ Janeiro. Rio de Janeiro-RJ, Brasil \\ (ricardo.steffen@gmail.com). \\ ORCID: 0000-0002-9733-5098

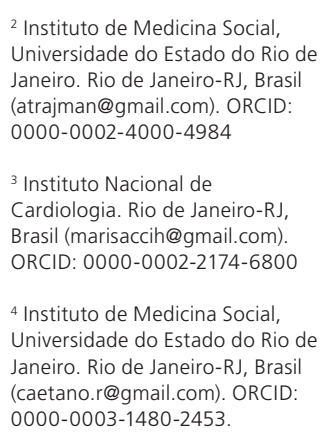

${ }^{4}$ Instituto de Medicina Social, Universidade do Estado do Rio de Janeiro. Rio de Janeiro-RJ, Brasil (caetano.r@gmail.com). ORCID: 0000-0003-1480-2453.

Recebido em: 08/03/2017 Aprovado em: 18/03/2018 Revisado em: 06/04/2018 
Nos últimos anos, campanhas nacionais promovidas por hospitais, sociedades médicas e outras organizações para estimular o rastreamento do câncer de próstata têm proliferado, em consonância com iniciativas mundiais conhecidas como Novembro Azul. Essas campanhas recomendam a utilização do toque retal acompanhado da dosagem sérica do antígeno prostático específico (PSA, da sigla em inglês correspondente a prostatic specific antigen) para homens a partir de faixas etárias definidas. A motivação subjacente seria a detecção precoce da neoplasia, com redução de sua mortalidade e das complicaçóes e impactos associados ao seu tratamento.

Trata-se de importante problema de saúde pública para os homens. As estimativas do Instituto Nacional do Câncer (INCA) sinalizam para a ocorrência de 61.200 casos novos deste câncer no ano de 2016, válido também para 2017, correspondendo a um risco de 61,82 casos/100.000 homens. Será a neoplasia mais incidente no sexo masculino em todas as regióes do país, sem considerar os tumores de pele não melanoma (BRASIL, 2015a).

O PSA foi introduzido nos anos 1980 como um marcador tumoral para detecção de recorrência e progressão da doença durante o tratamento. $\mathrm{O}$ teste tem baixas sensibilidade e especificidade e não existem evidências claras do limiar para indicar a biópsia. O limiar comumente utilizado ( $>4 \mathrm{ng} / \mathrm{mL}$ ) tem $70 \%$ de resultados falsopositivos (MULHEM et al., 2015). Elevaçóes do PSA precedem em 5 a 10 anos o aparecimento de doença clínica, mas seus níveis também aumentam em condiçôes benignas, como hiperplasia prostática benigna, prostatites e infecçóes do trato urinário inferior (DRAISMA et al., 2003).

A dosagem do PSA pode ser utilizada tanto em pacientes com sintomas (funcionando como método de diagnóstico), como em indivíduos assintomáticos, para fins de rastreamento. Seu valor benéfico no diagnóstico de indivíduos com suspeita clínica da doença é bem documentado na literatura.

O rastreamento da neoplasia de próstata não tem o objetivo de prevenir o câncer, apenas de realizar sua detecção precoce, antes do surgimento de sintomas da doença, o que poderia aumentar teoricamente a probabilidade de sucesso do tratamento, elevando a sobrevida ou melhorando a qualidade de vida (WILSON; JUNGNER, 1968). Seu uso no rastreamento populacional em indivíduos sem quaisquer sintomas é alvo de grande controvérsia nas publicaçôes científicas (DRAISMA et al., 2009). 
Para melhor se entender a controvérsia, dois conceitos relativos à detecção do câncer de uma forma geral precisam ser compreendidos. Um é o chamado lead time ou tempo de ganho, que corresponde ao tempo médio de ganho do diagnóstico proporcionado pelo rastreio (Figura 1). Em alguns estudos, o rastreamento parece aumentar falsamente a sobrevida, mas o que ocorre de fato é que os anos vividos "a mais" são aqueles antes do diagnóstico, sem adiamento do momento do óbito, o que é denominado de viés de antecipação (lead-time bias) (JANSEN et al., 2013). Esse tipo de viés é particularmente importante no caso de tumores indolentes e de crescimento lento (Figura 2). O diagnóstico precoce, nesses casos, apenas diminui a qualidade de vida, com a ansiedade gerada e as consequências indesejadas do sobretratamento (overtreatment) (HEIJNSDIJK et al., 2009).

Figura 1. Rastreamento para câncer de próstata e viés de antecipaçấo

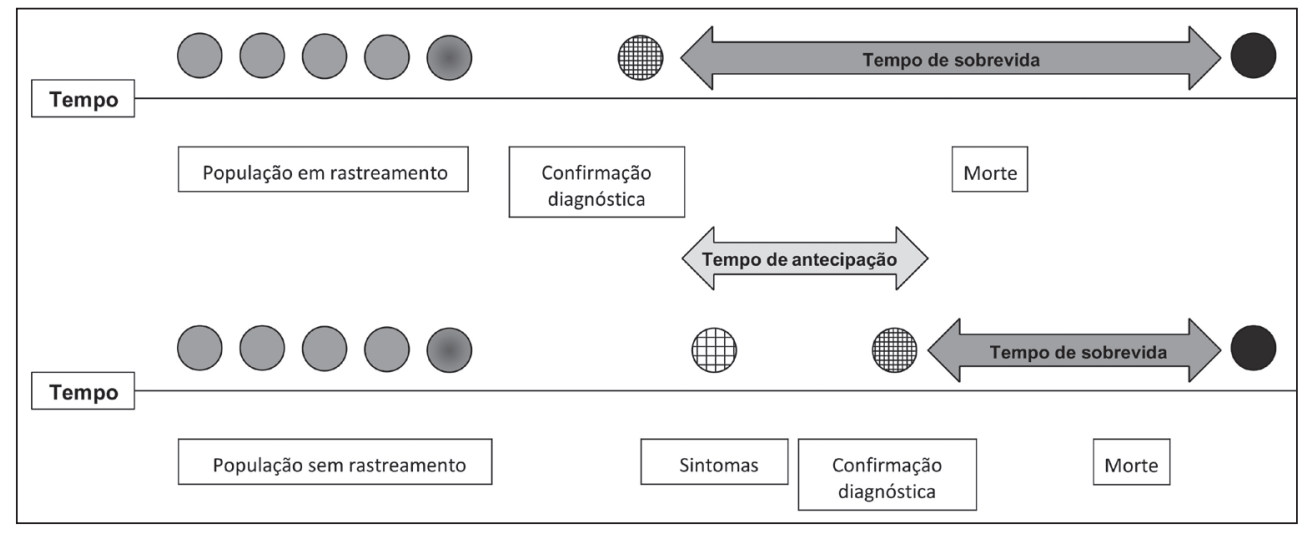

Fonte: Adaptado de Patz, 2000, p. 1.628. 
Figura 2. Rastreamento para câncer de próstata e características do tumor

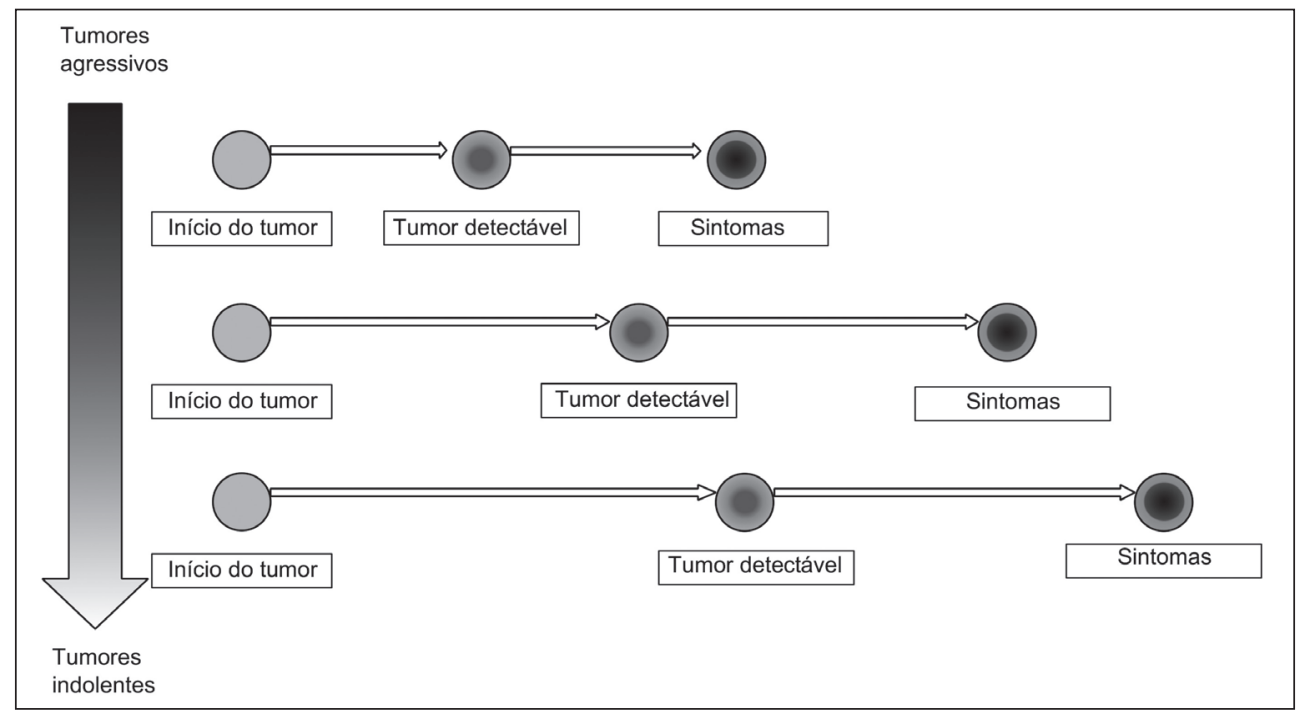

Fonte: Adaptado de Patz, 2000, p. 1.628.

Outro conceito fundamental é o de sobrediagnóstico (overdiagnosis), que se relaciona à detecção de lesóes que nunca seriam identificadas ao longo da vida dos indivíduos, que poderiam morrer por outras causas antes da manifestação clínica das neoplasias. Esse último aspecto tem sido elencado como preocupação importante no rastreamento dos cânceres de próstata, mama e pulmão (WELCH; BLACK, 2010).

Em estudos de autópsia, aproximadamente metade dos homens acima de 80 anos apresentam células malignas na próstata, mesmo sem terem apresentado sintomas enquanto vivos (JAHN et al., 2015). Ou seja, grande quantidade de tumores malignos localizados ou in situ identificados pelo rastreamento não interfeririam na sobrevida ou na qualidade de vida do paciente, porque seriam vencidos pela própria imunidade ou teriam uma evolução muito lenta. É difícil estimar a proporção de homens com sobrediagnóstico de câncer de próstata, que pode variar de 23\% a 66\% (DRAISMA et al., 2009).

Muitos dos pacientes assim diagnosticados são submetidos a procedimentos diagnósticos (biópsias) e 'curativos' (cirurgia, radioterapia) associados a elevadas morbidade e mortalidade, que seriam desnecessários. Isto resulta em sobretratamento, o qual também agrega custos indevidos (CHOU et al., 2011). 
Essa situação é agravada porque a dosagem do PSA em populaçôes assintomáticas não reduz de forma significativa a mortalidade. Metanálise publicada pela Biblioteca Cochrane em 2013 identificou cinco ensaios clínicos controlados randomizados que utilizaram dosagem de PSA, com ou sem a utilizaçáo de toque retal, envolvendo 341.342 participantes de 45-80 anos (ILIC et al., 2013). Não houve diferença na mortalidade específica por câncer prostático entre os pacientes randomizados para rastreamento e os controles (risco relativo [RR] 1,00, IC 95\% 0,86-1,17).

Nessa metanálise, um alto risco de viés foi identificado em três estudos que não apontavam para benefício na mortalidade (LABRIE et al., 2004; KJELLMAN et al., 2009; SANDBLOM et al., 2011). Os outros dois trabalhos incluídos, avaliados como tendo baixo risco de viés - European Randomized Study of Screening for Prostate Cancer (ERSPC) e US Prostate, Lung, Colorectal and Ovarian Cancer Screening Trial (PLCO) - trazem resultados conflitantes. O estudo PLCO, realizado com 76.693 participantes de centros americanos, não evidenciou qualquer benefício do rastreamento (ANDRIOLE et al., 2009). O seguimento de 13 anos desse estudo mostrou taxas de mortalidade cumulativa nos grupos intervenção e controle de, respectivamente, 3,7 e 3,4 mortes/10,0000 pessoas-ano, resultando em uma diferença não estatisticamente significante (RR 1,09, IC 95\% 0,87-1,36) (ANDRIOLE et al., 2012) Já o ERSPC envolveu 13.515 participantes de 55 a 69 anos, de oito países europeus, relatando reduçôes no RR de mortalidade específica por câncer no grupo submetido ao rastreamento: de $15 \%$ após nove anos de seguimento e de $21 \%$ após 13 anos, com redução absoluta de 0,11/1000 participantes-ano, representando uma morte evitada por câncer prostático a cada 781 indivíduos rastreados. A despeito desses números, os autores concluem que a quantificação e redução dos danos associados ao rastreamento deve ser considerada (SCHRÖDER et al., 2014).

Parte da discrepância entre os trabalhos parece ser explicada por diferenças nas populaçóes e tipo de estudo (diferentes critérios de elegibilidade, de esquemas de randomização e das estratégias de rastreamento e seguimento), bem como pela contaminação entre os braços (proporçóes altas nos grupos controle de homens submetidos a rastreamento) (SCHRÖDER; ROOBOL, 2010). Mais recentemente, um estudo que tenta conciliar os resultados divergentes dos ensaios ERSPC e PCLO, utilizando modelos matemáticos e de microssimulação, concluiu que o rastreamento reduz a mortalidade, mas que esta redução, embora seja de $20 \%$ a $30 \%$ em termos relativos, é muito pequena em termos absolutos: seria necessário 
rastrear 1.000 homens para prevenir uma única morte por câncer de próstata (TSODIKOV et al., 2017).

Ao mesmo tempo, é importante levar em consideração os danos que o rastreamento e as intervenções médicas podem causar. Níveis elevados de PSA frequentemente levam à biópsia prostática guiada por ultrassom, que se associa à dor, febre, hematúria, hematospermia, e hospitalização por prostatite e urosepse (ROSÁRIO et al., 2012). No ensaio ESPRC, um em cada cinco homens rastreados sofreram biópsia por resultado falso-positivo. Os tratamentos com o objetivo curativo incluem prostatectomia radical, radioterapia externa e braquiterapia, aos quais se associam elevados riscos de sangramento, incontinência urinária e disfunção erétil, sem contar com impactos psíquicos como ansiedade e depressão (DRAISMA et al., 2009).

Apesar dessas evidências, algumas sociedades médicas de outros países continuam recomendando a dosagem de PSA para todos os homens acima de 50 anos, enquanto outras instituiçóes adotam postura contrária a esse rastreamento (Tabela 1). Até recentemente, a Sociedade Brasileira de Urologia seguia essa indicação (NARDI, 2014), mas em publicação mais recente, datada de 2017, passou a recomendar que homens a partir de 50 anos conversem com seus urologistas sobre os exames de detecção precoce e que aqueles com fatores de risco, como histórico da neoplasia na família, negros e obesos, realizem essa consulta aos 45 anos (SBU, 2017).

Tabela 1. Resumo das recomendações das sociedades médicas e agências internacionais sobre o rastreamento para o câncer de próstata

\begin{tabular}{|c|c|c|c|c|c|}
\hline \multirow[t]{2}{*}{ País/Organização } & \multirow[t]{2}{*}{ Ano } & \multicolumn{3}{|c|}{ Idade recomendada } & \multirow{2}{*}{$\begin{array}{c}\text { Frequência do } \\
\text { rastreamento }\end{array}$} \\
\hline & & Início & Término & & \\
\hline $\begin{array}{l}\text { Austrália } \\
\text { National Health and } \\
\text { Medical Research } \\
\text { Council (NHMRC, } \\
2016)\end{array}$ & 2016 & \multicolumn{4}{|c|}{ Recomenda a decisão compartilhada } \\
\hline $\begin{array}{l}\text { EUA } \\
\text { United States } \\
\text { Preventive Services Task } \\
\text { Force (USPSTF, 2017) }\end{array}$ & 2017 & \multicolumn{2}{|c|}{$\begin{array}{l}55 \text { a } 69 \text { - Decisão } \\
\text { individualizada e } \\
\text { compartilhada }\end{array}$} & \multicolumn{2}{|c|}{$\begin{array}{l}\geq 70-\text { Nenhum } \\
\text { rastreamento }\end{array}$} \\
\hline
\end{tabular}

continua... 


\begin{tabular}{|c|c|c|c|c|}
\hline \multirow[t]{2}{*}{ País/Organização } & \multirow[t]{2}{*}{ Ano } & \multicolumn{2}{|c|}{ Idade recomendada } & \multirow{2}{*}{$\begin{array}{l}\text { Frequência do } \\
\text { rastreamento }\end{array}$} \\
\hline & & Início & Término & \\
\hline $\begin{array}{l}\text { EUA } \\
\text { American Academy } \\
\text { of Family Physicians } \\
\text { (MULHEM, 2015) }\end{array}$ & 2015 & \multicolumn{3}{|c|}{ Não recomenda o rastreamento } \\
\hline $\begin{array}{l}\text { EUA } \\
\text { American Cancer } \\
\text { Society (WOLF, 2010) }\end{array}$ & 2010 & $\begin{array}{l}50 \text { - risco- } \\
\text { padrão } \\
45 \text { - risco } \\
\text { aumentado } \\
40 \text { - risco } \\
\text { muito } \\
\text { aumentado }\end{array}$ & $\begin{array}{l}\text { Até expectativa de } \\
\text { vida do indivíduo } \\
\text { exceder } 10 \text { anos }\end{array}$ & $\begin{array}{l}\text { A cada } 2 \text { anos } \\
\text { ou anualmente } \\
\text { se PSA }>2.5 \mathrm{ng} / \\
\mathrm{mL}\end{array}$ \\
\hline $\begin{array}{l}\text { EUA } \\
\text { American Society of } \\
\text { Clinical Oncology } \\
\text { (BASCH et al, 2012) }\end{array}$ & 2012 & \multicolumn{3}{|c|}{$\begin{array}{l}\text { Expectiva de vida } \leq 10 \text { anos }- \text { Nenhum rastreamento } \\
\text { Expectiva de vida }>10 \text { anos }- \text { Decisáo individualizada e } \\
\text { compartilhada }\end{array}$} \\
\hline $\begin{array}{l}\text { EUA } \\
\text { American College } \\
\text { Physicians (QASEEM } \\
\text { et al., 2013) }\end{array}$ & 2013 & \multicolumn{3}{|c|}{$\begin{array}{l}\text { Náo recomenda rastreamento para homens com }<40 \\
\text { anos com risco médio; maiores de } 69 \text { anos e homens } \\
\text { com expectativa de vida } \leq 10-15 \text { anos } \\
\text { Homens de 50-69 anos - decisão individual informada } \\
\text { e compartilhada }\end{array}$} \\
\hline $\begin{array}{l}\text { EUA } \\
\text { American Urological } \\
\text { Association (AUA, } \\
2013 \text { ) }\end{array}$ & 2013 & 55 & 69 & A cada 2 anos \\
\hline $\begin{array}{l}\text { Canadá } \\
\text { Canadian Task Force }\end{array}$ & 2014 & \multicolumn{3}{|c|}{ Não recomenda o rastreamento } \\
\hline $\begin{array}{l}\text { Reino Unido } \\
\text { National Health } \\
\text { Service }\end{array}$ & 2015 & \multicolumn{3}{|c|}{$\begin{array}{l}\text { Programa de redução de riscos com a decisão } \\
\underline{\text { compartilhada }}\end{array}$} \\
\hline
\end{tabular}




\begin{tabular}{|c|c|c|c|c|}
\hline \multirow[t]{2}{*}{ País/Organização } & \multirow[t]{2}{*}{ Ano } & \multicolumn{2}{|c|}{ Idade recomendada } & \multirow{2}{*}{$\begin{array}{l}\text { Frequência do } \\
\text { rastreamento }\end{array}$} \\
\hline & & Início & Término & \\
\hline $\begin{array}{l}\text { Brasil } \\
\text { Sociedade Brasileira de } \\
\text { Urologia (SBU, 2017) }\end{array}$ & $2017^{*}$ & \multicolumn{3}{|c|}{$\begin{array}{l}>50 \text { anos - consulta e decisão compartilhada } \\
>45 \text { anos - consulta precoce para pacientes de risco } \\
\text { aumentado (raça negra ou com parentes de primeiro } \\
\text { grau com câncer de próstata) } \\
>75 \text { - exames realizados apenas para aqueles com } \\
\text { expectativa de vida acima de } 10 \text { anos }\end{array}$} \\
\hline $\begin{array}{l}\text { Brasil } \\
\text { Sociedade Brasileira de } \\
\text { Medicina de Família } \\
\text { (SBMF, 2016) }\end{array}$ & 2016 & \multicolumn{3}{|c|}{ Não recomenda o rastreamento } \\
\hline $\begin{array}{l}\text { Brasil } \\
\text { Instituto Nacional } \\
\text { do Câncer (BRASIL, } \\
\text { 2015a) }\end{array}$ & 2015 & \multicolumn{3}{|c|}{$\begin{array}{l}\text { Não recomenda a organização de programas de } \\
\text { rastreamento }\end{array}$} \\
\hline $\begin{array}{l}\text { Internacional } \\
\text { European Association } \\
\text { of Urology, } \\
\text { European Society } \\
\text { for Radiotherapy } \\
\text { \& Oncology e } \\
\text { International Society } \\
\text { of Geriatric Oncology } \\
\text { (MOTTET et al., } \\
\text { 2017) }\end{array}$ & 2017 & \multicolumn{3}{|c|}{$\begin{array}{l}\text { Oferecer rastreamento em base individualizada com } \\
\text { decisáo compartilhada em pacientes com expectativa } \\
\text { de vida de pelo menos } 10 \text { a } 15 \text { anos, nas seguintes } \\
\text { situaçôes de alto risco: } \\
\text { - > } 50 \text { anos } \\
\text { - > } 45 \text { anos e história familiar de câncer de próstata } \\
\text { - níveis de PSA superiores a } 1 \mathrm{ng} / \mathrm{ml} \text { aos } 40 \text { anos } \\
\text { - níveis de PSA superiores a } 2 \mathrm{ng} / \mathrm{ml} \text { aos } 60 \text { anos }\end{array}$} \\
\hline
\end{tabular}

Notas: * - última nota oficial da SBU, disponível em: http://portaldaurologia.org.br/destaques/notaoficial-2017-rastreamento-do-cancer-de-prostata/; ** _ Contrária à organização de programas de rastreamento populacional, porém recomendando compartilhar a decisão de rastreamento para detecção precoce com o paciente.

Como em países como a Austrália (NATIONAL HEALTH AND MEDICAL RESEARCH COUNCIL, 2016), Canadá (BELL et al., 2014) e Reino Unido (LOUIE, 2016), a U.S. Preventive Service Task Force (USPSTF, 2017), o Ministério da Saúde e o INCA não recomendam o rastreamento populacional para o câncer da próstata, baseados em evidências científicas de boa qualidade de que este produz 
mais dano que benefício. Indicam sua detecção precoce para homens que apresentem sintomas relacionados ao sistema urológico ou histórico familiar e reforçam que os riscos inerentes aos procedimentos devem ser apresentados e discutidos com o paciente (BRASIL, 2015b).

Está na hora de se repensar o papel do rastreamento no câncer de próstata e discutir os seus potenciais benefícios diante dos riscos associados ao viés de antecipação, sobrediagnóstico e sobretratamento. Os diversos pontos apresentados nesse manuscrito sugerem que é fundamental que seja empreendida uma discussão mais cientificamente rigorosa do que realmente deve ser feito para melhorar o manejo desse câncer no país. ${ }^{1}$

\section{Referências}

AMERICAN UROLOGICAL ASSOCIATION (AUA). Early Detection of Prostate Cancer: AUA Guideline, 2013. Disponível em: <https:/www.auanet.org/common/pdf/education/ clinical-guidance/Prostate-Cancer-Detection.pdf>. Acesso em: 15 fev 2017.

ANDRIOLE, G. L. et al. Mortality results from a randomized prostate-cancer screening trial. N Engl J Med, v. 360, n. 13, p. 1.310-9, 2009.

ANDRIOLE, G. L. et al. Prostate cancer screening in the randomized Prostate, Lung, Colorectal, and Ovarian Cancer Screening Trial: mortality results after 13 years of follow-up. $J$ Natl Cancer Inst, v. 104, n. 2, p. 125-32, 2012.

BASCH, E. et al. Screening for prostate cancer with prostate-specific antigen testing: American Society of Clinical Oncology Provisional Clinical Opinion. J Clin Oncol, v. 30, n. 24, p. 3.020-5, 2012.

BELL, N. et al. Recommendations on screening for prostate cancer with the prostate-specific antigen test. CMAJ, v. 186, n. 16, p. 1.225-34, 2014.

BRASIL. Ministério da Saúde. Instituto Nacional de Câncer. Estimativa 2016: incidência de câncer no Brasil. Rio de Janeiro: INCA, 2015a. Disponível em: <http:/www.inca.gov.br/ estimativa/2016/estimativa-2016-v11.pdf>. Acesso em: 30 jan 2017.

. Ministério da Saúde. Instituto Nacional de Câncer. Nota técnica conjunta nº 001/2015b.

Disponível em: <http://www2.inca.gov.br/wps/wcm/connect/tiposdecancer/site/home/ prostata/deteccao_precoce>. Acesso em: 30 jan 2017.

CHOU, R. et al. Screening for prostate cancer: a review of the evidence for the U.S. Preventive Services Task Force. Ann Intern Med, v. 155, n. 11, p. 762-71, 2011. 
DRAISMA, G. et al. Lead times and overdetection due to prostate-specific antigen screening: estimates from the European Randomized Study of Screening for Prostate Cancer. J Natl Cancer Inst, v. 95, n. 12, p. 868-78, 2003.

DRAISMA, G. et al. Lead time and overdiagnosis in prostate-specific antigen screening: importance of methods and context. J Natl Cancer Inst, v. 101, n. 6, p. 374-83, 2009.

HEIJNSDIJK, E. A. et al. Overdetection, overtreatment and costs in prostate-specific antigen screening for prostate cancer. Br J Cancer, v. 101, n. 11, p. 1833-8, 2009.

ILIC, D. et al. Screening for prostate cancer. Cochrane Database Syst Rev, n. 1, p. CD004720, 2013. JAHN, J. L. et al. The High Prevalence of Undiagnosed Prostate Cancer at Autopsy: Implications for Epidemiology and Treatment of Prostate Cancer in the Prostate-Specific Antigen-Era. Int J Cancer. v. 12, n. 137, p. 2.795-2.802, 2015.

JANSEN, R. J. et al. Quantifying lead-time bias in risk factor studies of cancer through simulation. Ann Epidemiol, v. 23, n. 11, p. 735-41, 2013.

KAWAKAMI, J. et al. Prostatitis and prostate cancer: implications for prostate cancer screening. Urology, v. 64, n. 6, p. 1.075-80, 2004.

KJELLMAN, A. et al. 15-Year follow up of a population based prostate cancer screening study. The Journal of Urology, v. 181, p. 1.615-1.621, 2009.

LABRIE, F. et al. 11-year follow-up of the 1988 Quebec prospective randomized controlled trial. Prostate, v. 59, n. 3, p. 311-318, 2004.

LOUIE, K. S. UK National Screening Committee. Screening for Prostate Cancer Review 2015 Update. Disponível em: <https://legacyscreening.phe.org.uk/policydb_download.php?doc.>, Acesso em: 15 jan 2017.

MOTTET, N. et al. EAU-ESTRO-SIOG Guidelines on Prostate Cancer. Part 1: Screening, Diagnosis, and Local Treatment with Curative Intent. Eur Urol, v. 71, n. 4, p. 618-629, 2017.

MULHEM E., et al. Prostate Cancer Screening. Am Fam Physician, v. 92, n. 8, p. 683-638, 2015.

NARDI, A. C. Câncer de próstata - diagnóstico. In: NARDI, A. C. (Ed.). Diretrizes Urologia - AMB. São Paulo: Câmara Brasileira de Livro, 2014.

NATIONAL HEALTH AND MEDICAL RESEARCH COUNCIL (NHMRC). Clinical practice guidelines: PSA testing and early management of test-detected prostate cancer. 2016. Disponível em: <http://www.cancer.org.au/health-professionals/clinical-guidelines/prostatecancer.html>. Acesso em: 15 fev 2017.

PATZ, E. F. JR.; GOODMAN, P. C.; BEPLER, G. Screening for lung cancer. $N$ Engl J Med. v. 343, n. 22, p.1.627-1.633, 2000. 
QASEEM, A. et al. Screening for prostate cancer: a guidance statement from the Clinical Guidelines Committee of the American College of Physicians. Ann Intern Med, v. 158, n. 10, p. 761-769, 2013.

ROSARIO, D. J. et al. Short term outcomes of prostate biopsy in men tested for cancer by prostate specific antigen: prospective evaluation within ProtecT study. BMJ, v. 344, p. d7.894, 2012.

SANDBLOM G., et al. Randomised prostate cancer screening trial: 20 year follow-up. BMJ, v. 342, p. d1.539, 2011.

SOCIEDADE BRASILEIRA DE MEDICINA DE FAMÍLIA E COMUNIDADE (SBMFC). Comunicado aos(às) apoiadores(as) da campanha Novembro Azul. Disponível em: <http:// www.sbmfc.org.br/media/Novembro\%20Azul.pdf>. Acesso em 27 jan 2017.

SOCIEDADE BRASILEIRA DE UROLOGIA (SBU). Nota Oficial 2017 - Rastreamento do Câncer de Próstata. 2017. Disponível em: <http://portaldaurologia.org.br/destaques/notaoficial-2017-rastreamento-do-cancer-de-prostata/>. Acesso em: 28 nov 2017.

SCHRÖDER, F. H. et al. Screening and prostate cancer mortality: results of the European Randomised Study of Screening for Prostate Cancer (ERSPC) at 13 years of follow-up. Lancet, v. 384, n. 9.959, p. 2.027-35, 2014.

SCHRÖDER, F. H.; ROOBOL, M. J. ERSPC and PLCO prostate cancer screening studies: what are the differences? Eur Urol, v. 58, n. 1, p. 46-52, 2010.

TSODIKOV A. et al. Reconciling the Effects of Screening on Prostate Cancer Mortality in the ERSPC and PLCO Trials. Ann Intern Med., v. 167, n. 7, p. 449-455, 2017.

U.S. PREVENTIVE SERVICES TASK FORCE (USPSTF). Task Force Draft Recommendations. Screening for Prostate Cancer. 2017. Disponível em: <https://www.uspreventiveservicestaskforce. org/Page/Document/draft-recommendation-statement/prostate-cancer-screening1>. Acesso em: 28 nov 2017.

WELCH, H. G.; BLACK W. C. Overdiagnosis in cancer. J Natl Cancer Inst., v. 102, n. 9, p. 605-613, 2010.

WILSON, J. M. G.; JUNGNER, G. Principles and practice of screening for disease. Geneva: World Health Organization, 1968. 164 p.

WOLF, A.M. et al. American Cancer Society guideline for the early detection of prostate cancer: update 2010. CA Cancer J Clin., v. 60, n. 2, p. 70-98, 2010.

\section{Notas}

${ }^{1}$ R. E. Steffen, A. Trajman, M. Santos e R. Caetano participaram igualmente da concepçáo e projeto do estudo, coleta e análise de dados, redação e aprovação da versão final do artigo. 


\section{Abstract}

Population screening for prostate cancer: more risks than benefits

Recently, numerous national campaigns promoted by hospitals, medical societies and other organizations have stimulated prostate cancer screening, in line with worldwide initiatives known as Blue November. These campaigns advise the use of rectal examination accompanied by dosage of serum prostate specific antigen (PSA) levels in defined age groups. The motivation would be the early detection of neoplasia, with reduction of mortality and complications and impacts associated with its treatment. The PSA dosage for screening purposes is highly controversial, since most of such tumors detected by screening are of slow progression and would not interfere with patient survival or quality of life. Population-based screening for prostate cancer is not recommended by numerous foreign institutions, including the National Cancer Institute in Brazil. The article discusses the risks and benefits associated with this type of strategy and reinforces concern about the inappropriate and indiscriminate use of screening for prostate cancer.

Keywords: prostatic neoplasm; mass screening; prostatespecific antigen; overdiagnosis. 